\title{
Prevention, Preparedness, and Wellness
}

James J. James, MD, DrPH, MHA

I would like to take this opportunity in this final issue of 2009 to underscore the insightful observations made by the newly appointed Assistant Secretary for Preparedness and Response, Dr Nicole Lurie, in recognizing the essential links between prevention, wellness, and preparedness. It is generally known and widely accepted that even though the United States spends more than $\$ 2$ trillion annually on health care, tens of millions of Americans continue to suffer from preventable injuries and diseases and major vulnerabilities still exist in the nation's preparedness to respond to public health emergencies.

What is not clearly seen are the links between a healthy population and an enhanced national health security. A 21stcentury health system must address these linkages and enhance and strengthen them by embedding preventive health practices as well as preparedness activities and response capabilities into national health reform efforts. Individuals and communities need to be able to prepare for and respond quickly to public health emergencies. National health responders need to be trained and educated and a culture of preparedness needs to be embraced by a resilient citizenry.

Prevention, preparedness, and wellness strategies are essential to help individuals and communities develop the resiliency and the coping skills to deal with individual and population-wide health challenges. Evidence supports various protective factors that may ameliorate risks to overall health. Much has been learned recently about the importance of "promotive" factors as simple as immunizations, exercise, good nutrition, adequate rest, healthy human interactions, and support from peers. It has also been shown that those with a personal preparedness plan are more likely to respond rather than become victims in disasters. Evidence also exists to support the fact although health care personnel recognize an obligation to respond, they do not feel adequately prepared to do so.

This journal has long advocated for a ready, willing, and able citizenry. Now, with the promise of health care reform we have an unparallel opportunity to define within its context a disaster health system built on a resilient and knowledgeable citizenry. A holistic approach is needed that takes into consideration appropriate developmental, cultural, and linguistic strategies. This can be accomplished by a sustained effort to increase individual and community resources for resilience, focusing on prevention of injury, disease, and disability through self-help measures and peer support, as well as through science-based community programs in education and risk communication.

Going forward, various prevention, preparedness, and wellness strategies can be integrated following an analysis of personal and community hazards, vulnerabilities, and risks. This includes providing health responders with educational and informational resources to

- Prevent the occurrence of death, injury, or illness in a disaster or public health emergency

- Mitigate the medical and mental health consequences of a disaster or public health emergency

- Minimize the effects of injury, disease, and disability among those with preexisting health conditions in a disaster or public health emergency

- Enhance personal and community capacity to prepare for, respond to, and cope with disasters and public health emergencies

- Fully integrate medical and mental health services into disaster preparedness and response plans (both pre- and postevent)

- Increase culturally and linguistically appropriate awareness programs and prevention-based services in communities to limit injuries and illnesses (both pre- and postevent)

- Increase access and availability of medical and mental health care and support systems for affected victims and populations (both pre- and postevent)

To accomplish all of this, stable and reliable funding streams are needed for core public health functions and preventive services, such as immunizations and screening, public health emergency preparedness, and promotion of physical activity, good nutrition, and smoking prevention. Coordinated action is needed to advocate wellness measures that can help individuals prevent, prepare for, and cope with the challenges of modern life and the multiple threats to health and safety. Based on existing research and experience, implementation of sound prevention, preparedness, and wellness approaches for the entire population must be a high priority at the national, state, and local levels. These approaches must be developed in culturally and linguistically competent ways for diverse populations with differing needs, and should be interwoven into the fabric of Healthy People 2020 and serve as a cornerstone of health system reform efforts-our national health security deserves nothing less.

\section{About the Author}

Dr James is Editor-in-Chief of Disaster Medicine and Public Health Preparedness and Director, Center for Public Health Preparedness and Disaster Response, American Medical Association.

Received and accepted for publication October 30, 2009

ISSN: 1935-7893 @ 2009 by the American Medical Association and Lippincott Williams \& Wilkins.

DOI: 10.1097/DMP.0b013e3181c8abd9 